\title{
Towards a Mobile Learning Environment using Reference Architectures
}

\author{
Maria Lydia Fioravanti \\ University of São Paulo \\ (ICMC/USP), São \\ Carlos (SP), Brazil \\ mlfioravanti@usp.br
}

\author{
Nemésio F. Duarte Filho \\ Federal Institute of São \\ Paulo (IFSP), São J. da \\ Boa Vista (SP), Brazil \\ nemesio@ifsp.edu.br
}

\author{
Lucas Bortolini Fronza \\ University of São Paulo \\ (ICMC/USP), São \\ Carlos (SP), Brazil \\ lfronza@usp.br
}

\author{
Ellen Francine Barbosa \\ University of São Paulo \\ (ICMC/USP), São \\ Carlos (SP), Brazil \\ francine@icmc.usp.br
}

\begin{abstract}
Mobile learning environments have emerged as a way to support the m-learning initiatives, providing benefits to learners, teachers and tutors. However, despite their relevance, the development of mobile learning environments present problems and challenges that must be investigated, especially with respect to the definition and adoption of architectural patterns. Motivated by this scenario, in this paper we discuss the development of a mobile learning environment, called ICMC MLE, following the precepts of a specific reference architecture for mobile learning. ICMC MLE was also evaluated through an experiment; the results showed a high level of satisfaction and convenience in relation to the use of ICMC MLE in real learning scenarios.
\end{abstract}

\section{Introduction}

Learning environments, together with the evolution of ubiquitous computing, have significantly contributed to the establishment of a new way of teaching and learning, known as mobile learning (mlearning) [1]. In short, mobile learning is characterized by the ability to promote a strong interaction among apprentices, teachers and tutors, assuring greater motivation, convenience and flexibility to the learning process.

By means of mobile devices (e.g., mobile phones, tablets, laptops, radio, tv, among others), teachers, tutors and learners can use the power of ubiquitous computing to contribute, participate and access learning materials at anytime and anywhere [2]. This is possible due to the interconnection between the web technology and the portability and integration of such devices, providing a high degree of communication and cooperation among their users.

Despite the benefits of mobile learning, it is still considered a new and incipient concept, having some limitations in its construction and use [2], [3] : (1) variable screen size; (2) limited energy (battery dependent); (3) transmission rates generally smaller than those of the fixed network; (4) adequacy to usability aspects; (5) and lack of architectural patterns, among others.

Considering the need of building mobile learning environments with quality, efforts for developing architectural patterns have become increasingly relevant in this context [1]. However, in spite of such efforts, there is a lack of a standardized set of models and reference architectures, specifically defined for the mobile learning domain and in agreement with educational issues.

Reference architectures emerged as an important mechanism in the definition of specific domains by means of modules and their relationships [3]. In general, a reference architecture refers to a special type of software architecture that captures the essence from a collection of architectures of systems in a given domain [3]. In addition, they can be considered as a knowledge repository of such domain. Among the benefits of reference architectures, we highlight the possibility of reuse of experiences through understanding of a specific domain.

Considering the growing need to build high quality, reliable and reusable mobile learning environments, efforts to establish architectural standards are even more relevant. Motivated by this scenario, in this paper we discuss the development of a mobile learning environment, called ICMC MLE, based on the proposal of a reference architecture. In short, the architecture defined intends to provide benefits with regard to domain understanding, establishment of a common vocabulary, architectural reuse, higher quality and reduced time spent in the development of such environments. The main findings obtained from the development and evaluation of ICMC MLE indicate that the proposed mobile learning environment has a high level of satisfaction and convenience in relation to learners and can be used in real learning contexts.

The remainder of this paper is organized as follows. In Section 2, we provide an overview of 
mobile learning and reference architectures. In Section 3 , related work is briefly presented. In Section 4, we describe the process used to propose reference architectures, their specialization and instantiation. In Section 5, we discuss the validation of ours ideas. Finally, in Section 6, we summarize our contributions and the main perspectives for future work.

\section{Background}

\subsection{Mobile Learning (m-learning)}

The rapid growth of information technologies along with the increasing flexibility in communications among users have provided new modalities of learning as well as innovative ways to deal with the limitations of traditional learning. For instance, due to the advent and evolution of technology, allied to the ubiquitous computing, a new modality of education based in mobile computing, referred to as mobile learning, has emerged [4].

According to Ozdamli and Cavus [5], m-learning refers to any kind of learning that occurs when the apprentice is not in a fixed place, or when he/she takes advantage of learning opportunities provided by mobile devices, thereby relating technological and mobility concepts.

The use of learning environments through mobile devices brings benefits that go beyond affordability, convenience and communication $[1,5]$. For example, with mobile devices apprentices can use the most different types of applications (e.g., text processors, photos, games), specific environments for learning, Web access, collaboration tools, social networks, among others.

Despite the benefits provided, due to the complexity and lack of architectural standardization regarding mobile learning environments, difficulties concerning the use, integration, maintenance and reuse of these environments are still common during their development. In this sense, the construction and adoption of mobile learning environments based in standards and guidelines can guarantee more adequacy for the educational practices.

The identification and understanding of guidelines for mobile learning environments is a complex task [6]. Different factors are involved in the development and adoption of such environments. Additionally to technical aspects, educational components, attributes of ubiquitous computing, criteria of mobile usability, among others, should also be taken into consideration.

\subsection{Reference Architectures}

According to Bass et al. [7], a software architecture can be described in terms of a structure that includes components, their external properties and the relationships among them, constituting a system abstraction. Its main role is to bridge the gap between requirements and implementation. In this sense, the idea is to support important issues of the project, such as the organization of the system as a composition of components, global control structures, communication protocols, the composition of design elements and the designation of the project components' features [8].

Reference architectures have been extensively investigated in the context of software architectures, providing a structure for the characterization of the software system functionalities of a given application domain [9], being an important artifact to be reused both in the development of new systems and in the evolution of existing systems. Its use has been explored in various fields (e-commerce systems, embedded systems, ubiquitous computing, robotic systems, among others).

\subsection{ProSA}

Aiming to systematize the establishment of reference architectures, we can consider ProSA

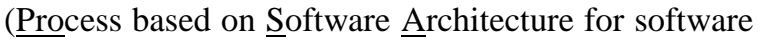
development) - an iterative process that involves design, specialization and architectural instantiation, aimed at incremental and evolutionary software development environments [8].

ProSA is a process focusing on software architectures, more specifically, reference architectures for developing software systems. Essentially, this process establishes the necessary steps for the construction and evaluation of reference architectures, as well as steps towards specialization and architectural instantiation, mainly targeting the reuse and thus increasing productivity in the development of software systems.

ProSA is composed of three processes, as shown in Figure 1 [10]. ProSA-RA consists of steps that allow the establishment, representation and evaluation of reference architectures. ProSA-S supports the specialization of the reference architecture. The specialization of reference architecture, or architectural specialization, refers to the refinement of a more general reference architecture of a domain in order to establish a more specific reference architecture. Finally, ProSA-I sets out the steps necessary for the instantiation of the reference architecture. The instantiation of the reference architecture, or architectural instantiation, refers to the creation of architectural instances from reference architectures. Architectural instance is the architecture of a particular software system. Then, after the establishment of the architectural instance, we start system design and implementation activities. 


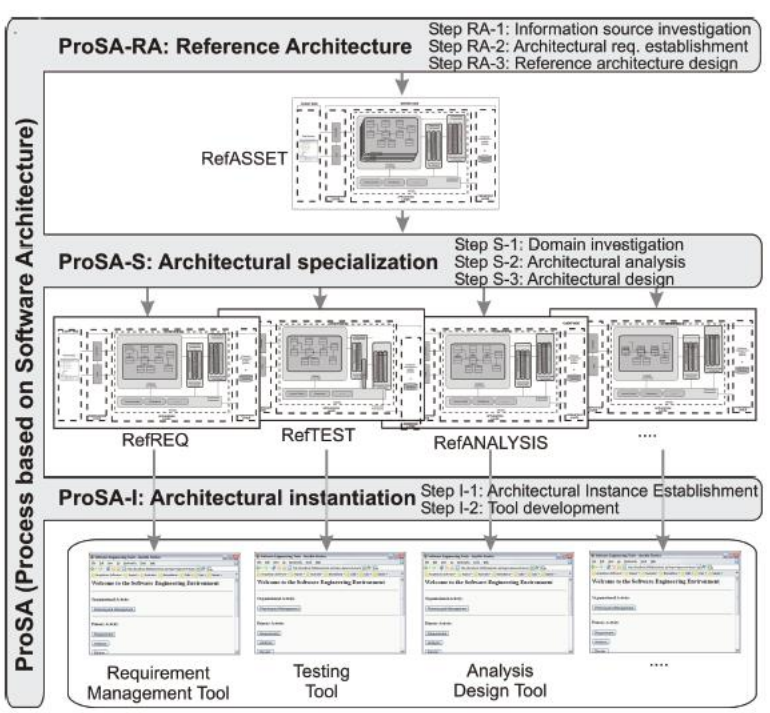

Figure 1. ProSA overview [10]

\section{Development of a Mobile Learning Environment by means of Reference Architectures}

In the context of learning environments and tools, one of the first works exploring more generic development structures was the AHAM (Adaptive Hypermedia Application Model) proposition, a reference model for hypermedia adaptive systems [11]. Later, other studies have begun to explore the concept of the reference architecture itself in the educational domain.

In this section, we present EDUCAR, a reference architecture for developing learning environments. Based on EDUCAR, we present Ref-mLearning, a reference architecture for developing mobile learning environments. We also present ICMC MLE, a mobile learning environment implemented based on RefmLearning. Both reference architectures and the mobile learning environment were developed according to ProSA.

\subsection{EDUCAR}

EDUCAR is an aspect-oriented reference architecture for developing learning environments [12]. The main goal of EDUCAR is to provide guidance for the architectural design of new versions of learning environments as well as promoting a better reuse, evolution and maintenance of the existing ones. EDUCAR has been constructed by following the activities prescribed by ProSA-RA.

3.1.1. RA-1: Information Sources Investigation. We began the establishment of EDUCAR by choosing a set of learning environments as information sources in this domain. Our selection was based on the following criteria: (i) the first initiatives on learning environments, such as WebCT/Blackboard; (ii) environments widely adopted, such as Moodle and Sakai; and (iii) environments with specific features, such as IWT (which explores the use of ontologies) and AdaptWeb (which addresses adaptive issues on learning).

Both proprietary and open source initiatives were investigated. At the end, 12 learning environments were considered. Experts' knowledge was also taken in account. Additionally, we conducted a systematic review [13] in order to identify related works addressing characteristics, functionalities and requirements of architectures of learning environments. We retrieved 60 research works and, then, based on the inclusion criterion defined, a subset of 40 works was considered for full reading and data extraction.

3.1.2. RA-2: Architectural Requirements Establishment. Based on the knowledge obtained from step RA-1, we could identify 13 categories of functionalities with respect to learning environments: Content, Learner's Assessment, Communication, Adaptation, Documentation, Course Coordination, System Administration, Storage, Standards Adequacy, Multilanguage, Interface, Interaction Mechanisms, and Access Mechanisms.

Each category was subcategorized and, for each of them, a set of functionalities was identified, from which 123 system requirements for learning environments were identified. Then, we conducted a detailed analysis of such requirements to identify the architectural requirements. The 123 system requirements were mapped into a set of 18 architectural requirements.

From the architectural requirements, we could determine the main concepts related to the learning environments domain and, then, identify which of these concepts had crosscutting characteristics. At the end, 13 concepts were established, one of them presenting a crosscutting characteristic (personalization).

3.1.3. RA-3: Reference Architecture Design. The proposition of EDUCAR was based on well-known and consolidated architectural styles of interactive systems and web systems found in the learning environments previously analyzed: the architectural pattern MVC and the three-tier architecture. To adequately represent EDUCAR, we built its architectural views (module view, runtime view and deployment view) using UML. For the sake of space, only the module view (Figure 2) is discussed herein. 


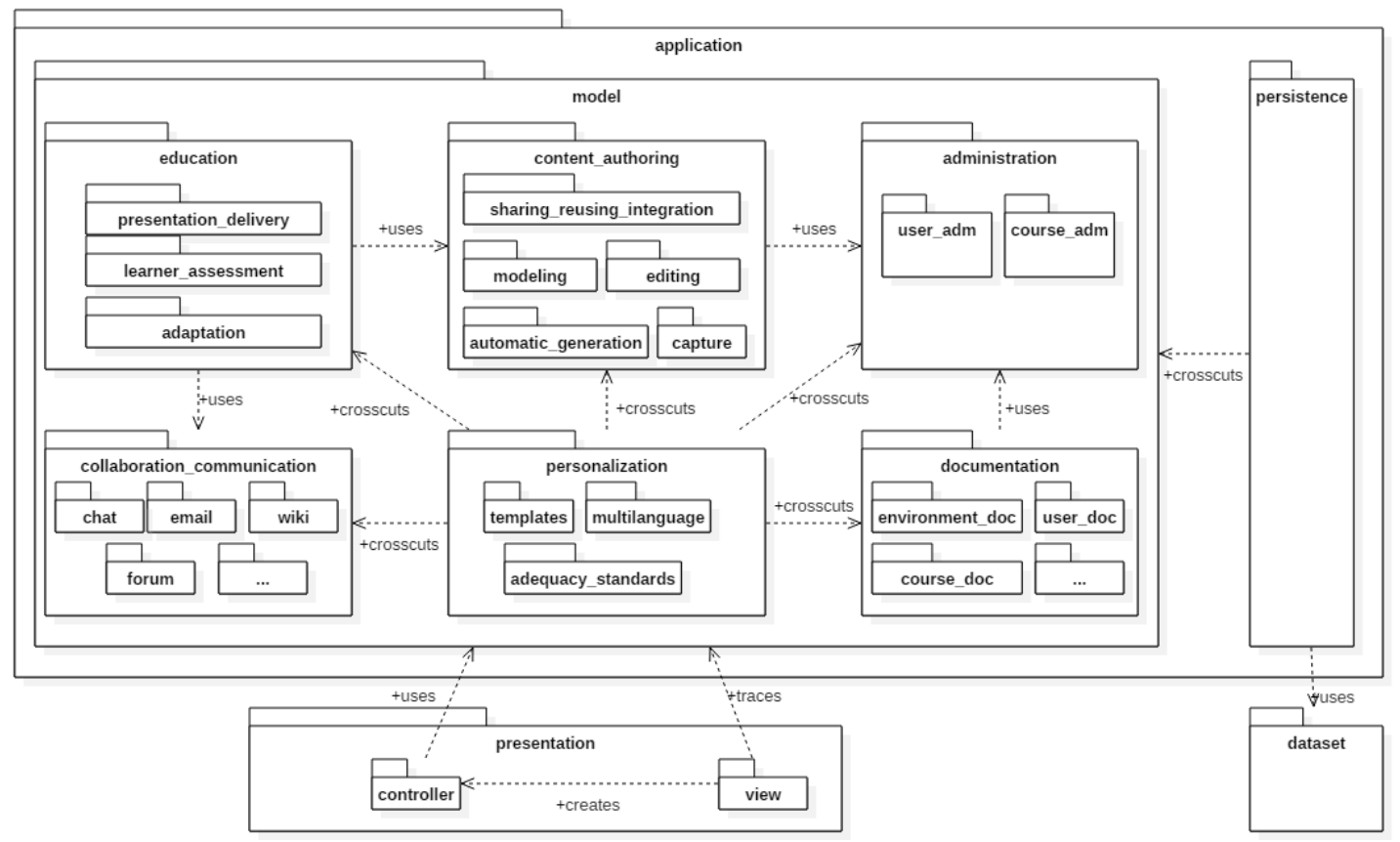

Figure 2. EducAR: module view

The module view is composed of three tiers/layers: persistence, presentation and application. The persistence layer corresponds to the set of data that needs to be stored by the learning environment: a database, a repository or a file system can be used.

The presentation layer refers to server side modules, which are responsible for the user interface presented in client side. This layer is composed of: (i) the Controller element, which processes events (typically user actions) and invokes the functionalities implemented by the Model element; and (ii) the View element, which contains the user interface.

The application layer contains the Model element, which aggregates the functionalities related to the core of learning environments. This layer comprises six modules: content_authoring, education, collaboration_communication, administration, documentation and personalization.

The content_authoring module is one of the core modules of EDUCAR, being responsible for the development of educational content (i.e., materials and assessments). Issues addressed in this module are related to: (i) structuring and modeling of content: involves the identification and representation of concepts and their inter-relationships, and instructional activities (e.g., exercises, practical assignments, and lab tasks); (ii) editing of content: involves the creation of documents and media (e.g., texts, slides, images, and videos); (iii) automatic generation of content: relevant when the content is represented in a machine-readable format; (iv) sharing, reuse and integration of content: they refer to the use of domain ontologies, dictionaries of terms, glossaries, among others, as supporting mechanisms to the development and evolution of content; and (v) capture of content: refers to the capture and storage of discussions and experiences that occurred during classes and later integration and synchronization of the multiple streams of information captured (e.g., audio, video, and notes).

The education module is also a core module of EDUCAR, being responsible for the presentation and delivery of educational content as well as the learners' assessments. It also covers issues related to the content adaptation. According to parameters such as background, objectives, interests and learning profile of each learner, different ways of structuring and navigating for the same content is established.

The collaboration_communication module gathers supporting tools for synchronous and asynchronous communication (e.g., chats, web conferences and emails) and for collaborative work (e.g., wikis and forums).

The administration module covers administrative issues, focusing on the management of users and courses. Regarding the management of users, it addresses issues of authentication and establishment of access levels to the users, as well as inclusion, exclusion and update of the users' information. Reports of learners' performance, participation and frequency are also considered. In terms of course management, the module covers topics such as course 
inclusion, exclusion and update, generation of statistics, and course backup, among others.

The documentation module is responsible for providing mechanisms for the management and storage of documents. Thus, documentation on the learning environment (e.g., help and FAQ), users and courses (e.g., objectives, lesson plans, schedule of classes and course FAQ) must be considered. Other types of documentation and/or relevant information to the environment can also be considered.

Finally, the personalization module establishes mechanisms for the creation and use of templates, support of multilanguage and adequacy to standards (such as IMS, SCORM and LOM). Personalization was classified as a crosscutting concern. So, this module must encapsulate a crosscutting concern and, therefore, it is an architectural aspect. As a crosscutting concern, it affects all other modules. Indeed, the functionalities implemented in this module impact other modules, changing their behavior to address functionalities related to personalization.

To compose an integrated learning environment, communications among modules/packages must also be established. Regarding relationships among the packages/modules, the module content_authoring communicates with the module education to make the content available to learners. It also communicates with the module administration to get information about the users' access levels for determining, for example, if a given user is allowed to create content in some course. The module education communicates with the module collaboration_communication since collaborative and communication tools can also be used to support the learning activities. Finally, the modules administration and documentation communicate each other since documentation is also responsible for documenting the information about users and courses managed by the module administration.

Aiming to promote separation of concerns in the learning environments built from EDUCAR, each module in the application layer was designed as separated as possible, enabling that each one can be designed and implemented as an independent tool (or subsystem). They can be further aggregated in a learning system, composing an integrated environment. Particularly, the module personalization, which aggregates a crosscutting concern, can be also developed as an independent tool. Additionally to the modules in the package model, we have foreseen the package crosscutting_services. It is composed of other architectural aspects that automate services considered crosscutting concerns, such as persistence and access control.

\subsection{Ref-mLearning}

Following the ProSA steps, during ProSA-S stage EDUCAR was specialized, resulting in a specific reference architecture, entitled Ref-mLearning, which focus on mobile learning context. Ref-mLearning also incorporates aspects of service-oriented architecture (SOA) [14], ensuring guidelines for reuse and interoperability of educational environments.

3.2.1. S-1: Domain Investigation. We began the establishment of Ref-mLearning complementing EDUCAR's information through specific domains investigation: mobile learning and SOA. During the domain investigation, the idea is to get considerable knowledge about the target domain. This knowledge is a basis for the establishment of the architectural requirements. In our case, through a semi-structured revision, two groups of information sources were defined, based on their relevance in the context of m-learning environments and SOA: (1) Concrete Architectures for Mobile Learning Environments; and (2) Service-Oriented Architectures. A complete description of the information sources used, as well as their classification according to the application domain of each one, can be found at Duarte Filho and Barbosa's work [15].

3.2.2. S-2: Architectural Analysis. The results of the domain investigation, complementary to EDUCAR, were mapped into a new set of architectural requirements for service-oriented m-learning environments. In short, the set of requirements was divided into two distinct groups: Architectural

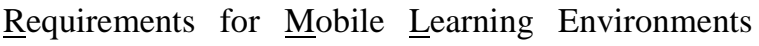
(AR-ML) and Architectural Requirements specific to SOA (AR-S).

Table 1 presents some of the architectural requirements identified. In total, 22 architectural requirements were established, benefiting the identification of needs and architectural concepts in the domain of mobile learning applications and SOA. The complete list of architectural requirements is available in Duarte Filho and Barbosa's work [16].

3.2.3. S-3: Architectural Design. Ref-mLearning was defined by four architectural views: (1) General View; (2) Module View; (3) Runtime View; and (4) Deployment View. For the sake of space, only the general view is discussed herein. The other views can be found in Duarte and Barbosa's work [15]. Figure 3 shows the general view of Ref-mLearning, which was defined in accordance with the architectural requirements previously discussed. The application layer presents specific modules of the m-learning domain. 
Table 1. Architectural Requirements- Ref-mLearning

\begin{tabular}{|c|l|c|}
\hline Identification & \multicolumn{1}{|c|}{ Description } & Group \\
\hline 1 & $\begin{array}{l}\text { The architecture should support/enable the development of m-learning environments that allow synchronous and } \\
\text { asynchronous communication. }\end{array}$ & $\begin{array}{l}\text { AR-ML } \\
\text { context, ensuring adaptation to the user's context in relation to physical, social and timing issues, among others. }\end{array}$ \\
\hline 2 & $\begin{array}{l}\text { The architecture should support/enable the development of m-learning environments that provides features to improve the } \\
\text { social interaction among users. }\end{array}$ & AR-ML \\
\hline 3 & $\ldots$ & $\begin{array}{l}\text { The architecture should enable the development of scalable learning environments, capable of incrementally evolving } \\
\text { through the addition of new services. }\end{array}$ \\
\hline 21 & $\begin{array}{l}\text { The architecture should allow that educational tools implemented in different programming languages and under different } \\
\text { platforms can be easily integrated. }\end{array}$ \\
\hline 22
\end{tabular}

Furthermore, it incorporates elements related to SOA, enabling greater reuse and interoperability. The elements described in this vision can be implemented
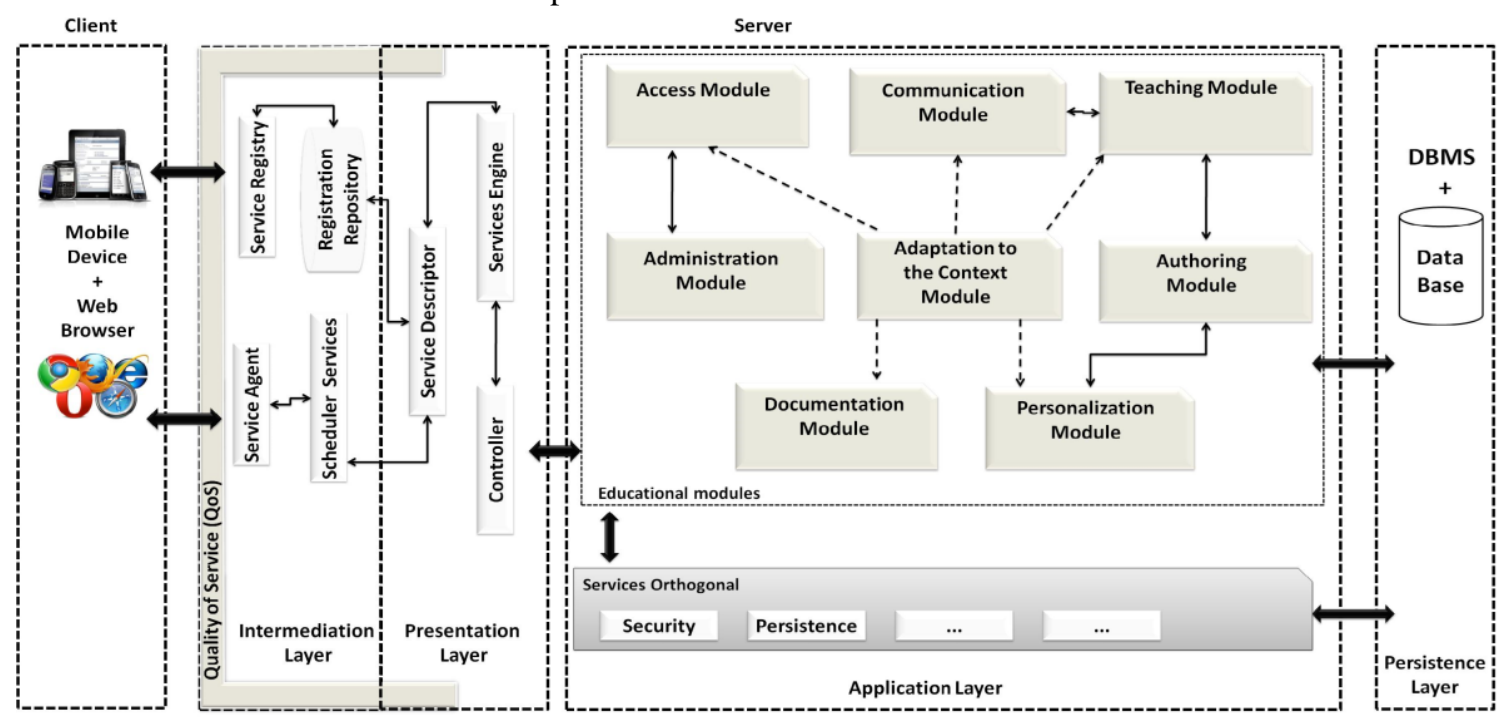

Figure 3. Ref-mLearning: general view

Presentation Layer: this is a server-side layer, whose primary task is to receive information requests from the client application, and to perform the visual presentation of information on the learning activities. This layer should analyze the original data and request the appropriate style information (e.g., XML). In addition, it should send the information in an appropriate style to the application server, transferring again for the requesting client of the information. To provide more compatibility with aspects of SOA and to increase interoperability and reuse of resulting applications, three modules were defined into RefmLearning: (i) Service Descriptor: defines the data types used in the request of functionalities; (ii) Requests Controller: is responsible for orchestrating the execution of other modules, ensuring services synchronization; and (iii) Services Engine: processes the services requests.

Quality of Service Layer (QoS): every service, being consumed or produced, must be in accordance with quality requirements. In the educational context, using technologies and languages that are most appropriate to their implementation. 
developed focusing on modularity and cohesion, to be used in different environments, therefore increasing the reuse. This layer consists of modules similar to EDUCAR, having specific modules to the mobile learning domain, as follows:

Adaptation to the Context Module: m-learning environments must be able to automatically detect all information related to the context of the users and tutors (e.g., place, time and, in some cases, physical conditions). This module is fundamental for detecting and recording the learner's current situation in the learning environment. The idea is to provide the teachers and tutors a greater understanding and knowledge about the apprentices.

Collaboration/Communication Module: this module defines the type of communication used by the mobile learning environment. The communication can be performed asynchronously or synchronously by means of a mobile device using the phone services. In general, this module allows that users of the environment determine the way of communication, e.g., SMS, MMS, speech interface or only keyboard.

\subsection{ICMC MLE}

Based on Ref-mLearning architecture, we developed a prototype entitled ICMC Mobile Learning Environment ${ }^{1}$. The main goal was to evaluate the viability of practical application of the proposed architecture.

3.3.1. I-1: Architectural Instance Establishment. To make the application accessible by multiple platforms and multiple mobile devices, ICMC MLE was developed as a web application instead of a native Android or iOS app. This approach was possible by using a responsive, mobile-first front-end framework, that allowed the application to be used on smartphones, tablets and even on desktops with large screens.

Since Ref-mLearning is a service-oriented architecture (Figure 4), one of the goals of the prototype development was to build educational web services that could be consumed by ICMC MLE and by other applications. To accomplish this, ICMC MLE was composed by two applications. One application was responsible for managing users, either students, tutors or teachers, managing their permissions, managing courses and hosting course files. In addition, this application was responsible for consuming third party services and implemented services as well. The other application was responsible for hosting all implemented educational web services. Even these web services being built as one single application, sharing the same database, it is worth saying that each one of them works independently.

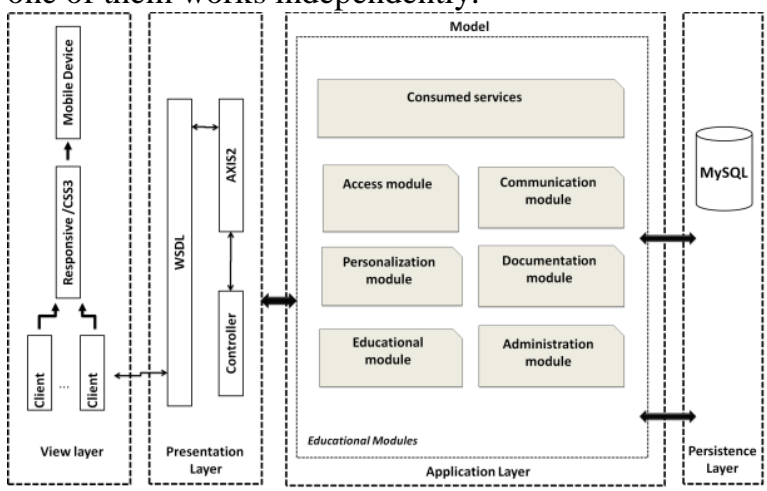

Figure 4. ICMC MLE concrete architecture

3.3.2. I-2: Tool Development. By following the RefmLearning service-oriented architecture, we were able to develop a modularized mobile learning environment and to provide reuse of implemented web services, i.e., these web services can be used by others in order to build their own mobile learning environments. Among the educational services implemented, we can point out Quiz, Score board and Attendance board. All functionalities, implemented either in the usual way or as service, and also the consumed services, can be seen on Table 2 .

Figure 5 shows two sample views of ICMC MLE: (a) Welcome Page, which is also the user Authentication Page; and (b) Microblog feature, which replaces a traditional forum. Microblog's idea is to provide a better communication and collaboration among students, teachers and tutors in the context of mobile learning, easing practices and educational activities.

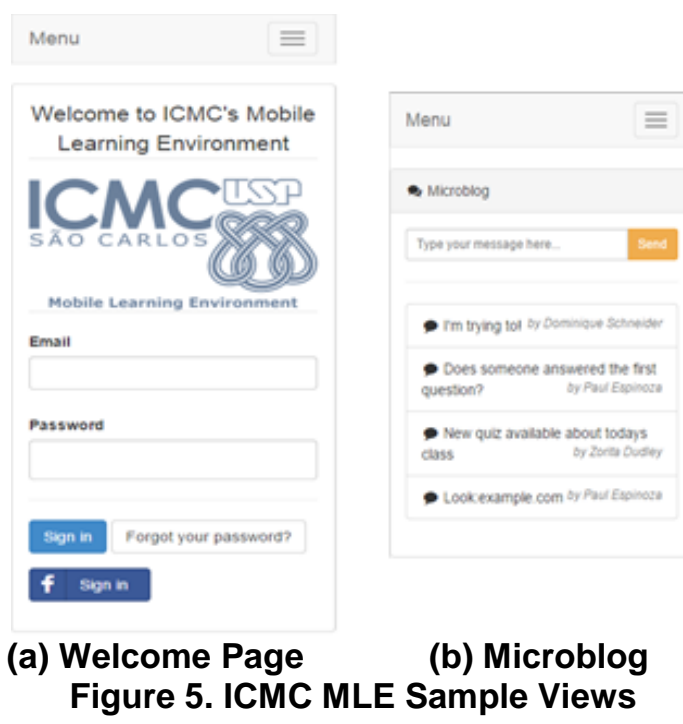

\footnotetext{
${ }^{1}$ http://www.labes.icmc.usp.br/ mle/
} 
Table 2. Prototype functionalities and services (developed and consumed)

\begin{tabular}{|c|c|c|}
\hline Functionalities Implemented (in the usual way) & Third party services & Developed as a service \\
\hline Educational content and data repository & Password recovery & Score board \\
\hline Message trading between tutors and students & Facebook sign in & Email notification \\
\hline New user sign up & Address autocompleting by ZIP code & Wiki \\
\hline Course management & SMS/MMS services & Attendance board \\
\hline Microblog for collaboration & & Quiz service \\
\hline
\end{tabular}

\section{Validation}

In this section, the authors have given greater importance to validate the ICMC MLE, since validations related to the reference architectures EducAR and Ref-mLearning were performed in earlier times. We emphasize that the reference architectures were theoretically validated by means of comparison with the reference model RAModel [15] and reviews by experts in the field [12]. The validation was also carried out through the application of evaluation checklists using the knowledge of experts in the field, ensuring greater theoretical and structural validation concerning organization and elements present in the reference architectures.

In order to validate the ICMC MLE environment we have conducted an experiment on a real learning scenario. The experiment protocol was defined based on GQM method [17], according to the following:

- Object of study: the analysis and acceptance of ICMC MLE use in the learning context, considering undergraduate students;

- Purpose: ICMC MLE environment evaluation;

- Focus: students' attitudes towards educational activities execution;

- Perspective: academic;

- Context: undergraduate students.

The research hypothesis defined was:

H1: ICMC MLE can be used as an m-learning environment to support undergraduate courses.

Dependent Variables: Satisfaction of participants; Perception of the participants; and Percentage of fulfilled educational activities.

Independent Variables: Suggested activities; Experience of students; Learning environment (mobile / traditional); Teacher and subject of the course; and Work environment.

For the sake of space, the complete protocol is available

http://www.labes.icmc.usp.br/ mlfioravanti/hicss201 6/protocol.pdf.

\subsection{Procedures}

The procedures performed during the experiment were: (i) planning; (ii) training; (iii) execution; and (iv) analysis.

Planning: At this stage, the authors defined objectives, questions, goals, procedures, variables, threats to validity, among others. It is noteworthy that at the end of planning definition, a written document was sent to a specialist in experimental software engineering, who reviewed and pointed out improvements.

Training: This phase aimed to ensure that participants were familiar with the environment, activities, artifacts and methods considered in the experiment. The training consisted of: (i) demonstration of ICMC MLE, exemplifying its educational features and practical application; and (ii) execution of a similar activity, showing the participants what would be the difficulty level, thus being able to address some questions to the instructor of the experiment. The authors also conducted pilot tests (data obtained from the tests were not considered in the results).

Execution: The whole experiment was conducted with undergraduate students of the Computer Science course at USP (University of São Paulo), attending Software Engineering classes. A total of 55 students participated, aiming to evaluate the practical application of ICMC MLE in relation to support daily basis educational activities.

Each student had one week to perform the educational activities with the support of ICMC MLE. Students going through the training received a printed form with the roadmap of activities to be performed and a brief description of them.

Throughout the experiment, the learners performed three different types of tasks related to the educational context: (i) access to documents and learning materials; (ii) collaboration and communication between learners; and (iii) secure the knowledge and feedback of knowledge acquired.

Task 1 aimed to make learners access the course material via ICMC MLE to verify if such traditional activity in a virtual learning environment could be easily carried out via a mobile device in a mobile learning environment.

Task 1 - Reading of the text / article A .pdf file will be uploaded into the repository of the educational environment, ICMC MLE.

Theme of the article: "An Approach to Quality Assessment of Web Application"

Task 2 was related to the ability of ICMC MLE to provide easy and flexible communications to its users. Being a mobile learning environment, it uses a microblog to perform communications. It was 
expected that, in this task, learners could use intuitively the microblog (similar to a social network), performing a short discussion of the subject topics.

\section{Task 2 - Discussion on Microblog}

After reading the text (Task 1), do:

"Based on the attributes of internal and external quality of ISO 9126, indicate which criteria are more difficult to evaluate in practice. Explain. Use your programmer / developer experience to discuss"

Note: A dedicated forum will be set up at ICMC MLE for conducting this discussion.

Task 3 aimed to assure that ICMC MLE can secure the knowledge by the students and also provide a feedback about their successes and failures. For such a need, the learner was supposed to perform a quiz with four questions in the environment, and receive a feedback on his/her mistakes and successes.

Task 3 - Knowledge Fixation

Students, after completing the previous tasks, must answer four questions related to the proposed theme.

The questionnaire will be available on ICMC MLE (Located in the Quiz of the class - Software Engineering). Students will have an immediate feedback from their rights and wrongs, along with justifications.

Analysis: In this phase, data were analyzed to provide assertive results and conclusions. It is noteworthy that in the data analysis, only data related to the execution phase were indeed considered.

\subsection{Results Analysis}

After performing the experiment, students answered an online questionnaire, which allowed us a full analysis of the data. In general, the learners highlighted positive aspects related to the ease of use and convenience of a mobile device; features that directly support educational activities related to reporting, collaboration / communication and educational feedback. As negative aspects, the learners showed a lack regarding: (i) restructure the microblog into categories and enable research in topics; (ii) allow private messages on microblog; and (iii) functionality for auto complete (messages).

According to Figure 6, the students were very satisfied or satisfied $(80,5 \%)$ with ICMC MLE. Few students $(17,1 \%)$ considered the environment regular and only $2,4 \%$ of them were unsatisfied. We can reassure students' satisfaction when we analyze their intention to use ICMC MLE again.

Analyzing Figure 7 (a), we notice that task 1 was easily performed and noticed by learners through the mobile learning environment. It is worth mentioning that no one found difficulties to accomplish the task and $74 \%$ of the students completely agree with the easiness of use to do it. Most reviews on the mechanisms and functions of this activity were positive, suggesting that the environment provides greater convenience and flexibility.

Regarding task 2, the results showed us a different scenario. In Figure 7 (b), we can notice that a small percentage $(10 \%)$ of participants indicated a partial disagreement on the easiness of performing such activity. Despite the disagreement, it is worth to highlight some comments provided, indicating a lack of familiarity with this type of communication via microblogging. Even though it is similar to a social network, many students were not familiar with microblog as they were with traditional forums.
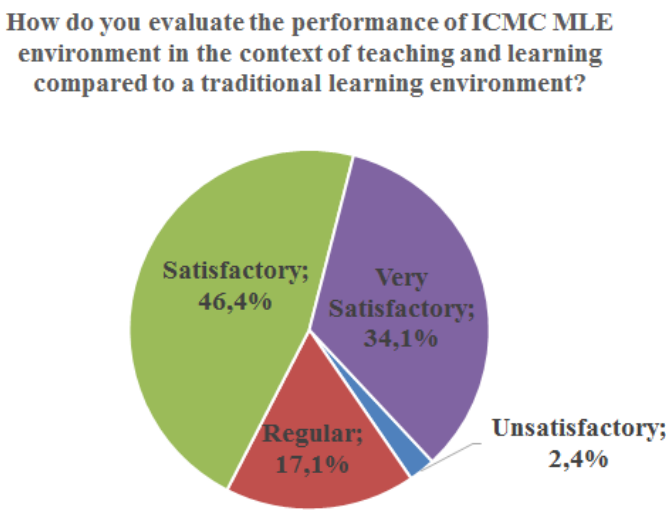

\section{Figure 6. Satisfaction with ICMC MLE compared to traditional environments}

Finally, from Figure 77 (c), we can see that task 3 was easily performed and highly accepted by students. Only $2 \%$ of learners showed a partial disagreement. However, several positive reviews were received, emphasizing the relevance of having a functionality that allows to incorporate the feedback.

From the obtained results, we can conclude that ICMC MLE had a high acceptance by the apprentices, who mentioned more convenience of use to accomplish educational activities. Overall, ICMC MLE was more dynamic and flexible, especially allowing students to access it anywhere and at any time through a mobile device with web access. The built-in features, like an e-learning environment, were adapted to the context of mobile learning, ensuring greater collaboration among students.

Regarding the threats to validity of the experiment, we highlight: (i) the reduced number of participants; (ii) the students' experience in relation to learning environments; and (iii) the non-participation of teachers and tutors during the experiment. In this context, in order to ensure greater validation to ICMC MLE, other experiments have been planned and will be conducted in short time. 


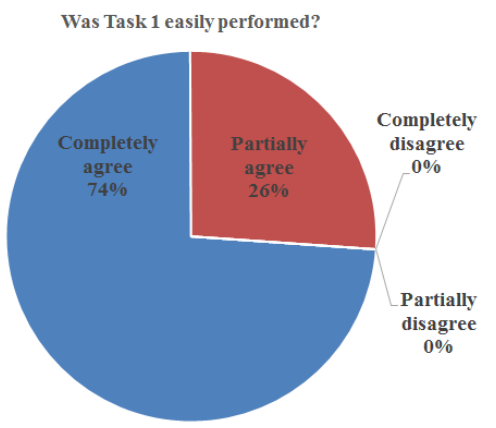

(a) Task 1

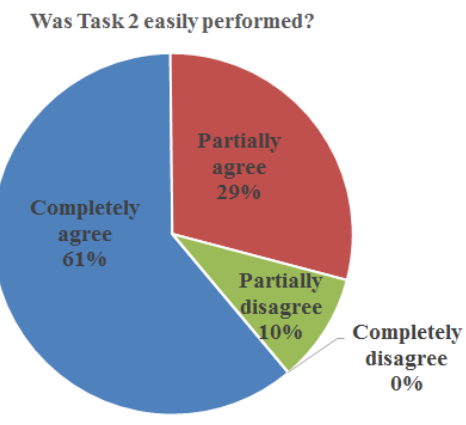

(b) Task 2

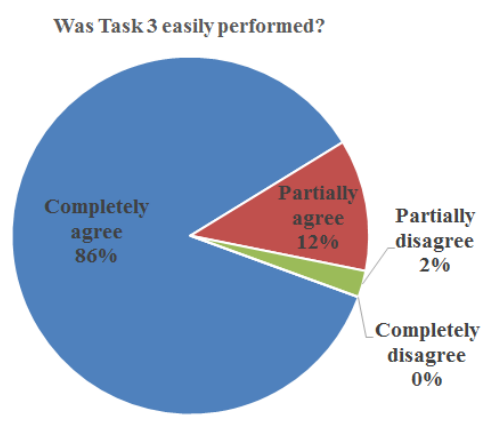

(c) Task 3

Figure 7. Easiness on accomplishing tasks

\section{Conclusions and Future Work}

In this paper, we described two reference architectures, one (Ref-mLearning) specialized from the other (EDUCAR), and also the establishment of an architectural instance and a system design and implementation (ICMC MLE). The main contribution of each work (separately or together) is to provide mechanisms to facilitate the design, development and/or maintenance of learning environments.

As future work, we point out the possibility of instantiating these reference architectures to others domains, such as learning through simulations, industrial training, assistive wearables and augmented reality. We also intend to perform other experiments with an improved version of ICMC MLE (i) having a greater number of students; (ii) focusing on teachers and tutors' activities; (iii) comparing learning in different mobile learning environments; among others. Such experiments have already been planned and will be conducted soon.

As a final remark, we highlight that knowledge about any domain emerges, evolves and consolidates over time. Reference architectures must encompass this new knowledge and must also be continually updated. So, EDUCAR and Ref-mLearning must also be continually evolved, inserting these new types of knowledge in order to not deteriorate.

\section{Acknowledgements}

The authors acknowledge Brazilian funding agencies - FAPESP (Processes 2013/07375-0 and 2014/03389-9), CAPES (Procad 071/2013) and CNPq - for their support.

\section{References}

[1] S. Martin, J. Peire, and M. Castro, "M2Learn: Towards a homogeneous vision of advanced mobile learning development," in Education Engineering (EDUCON), 2010 IEEE, 2010, pp. 569-574.
[2] A. C. Jones, E. Scanlon, and G. Clough, "Mobile learning: Two case studies of supporting inquiry learning in informal and semiformal settings," Comput. Educ., vol. 61, pp. 21-32, 2013.

[3] D. C. Schmidt, M. Stal, H. Rohnert, and F. Buschmann, Pattern-Oriented Software Architecture, Patterns for Concurrent and Networked Objects, vol. 2. John Wiley \& Sons, 2013.

[4] E. I. Basaeed, J. Berri, M. J. Zemerly, and R. Benlamri, "Webbased context-aware m-learning architecture," Int. J. Interact. Mob. Technol., vol. 1, no. 1, pp. 5-10, 2007.

[5] F. Ozdamli and N. Cavus, "Basic elements and characteristics of mobile learning," Procedia-Social Behav. Sci., vol. 28, pp. 937-942, 2011.

[6] E. Baran, "A Review of Research on Mobile Learning in Teacher Education.," Educ. Technol. Soc., vol. 17, no. 4, pp. 17-32, 2014

[7] L. Bass, P. Clements, and R. Kazman, Software Architecture in Practice, 2nd ed. 2003.

[8] E. Y. Nakagawa, F. Oquendo, and J. C. Maldonado, "Reference architectures," Softw. Archit. 1, pp. 55-82, 2014.

[9] J. Herzog, "Software Architecture in Practice Third Edition Written by Len Bass, Paul Clements, Rick Kazman.," ACM SIGSOFT Softw. Eng. Notes, vol. 40, no. 1, pp. 51-52, 2015.

[10]E. Y. Nakagawa, "Uma Contribuição ao Projeto Arquitetural de Ambientes de Engenharia de Software," phdthesis, ICMC/USP, São Carlos, SP, 2006.

[11] H. Wu, "A reference architecture for adaptive hypermedia applications," Eindhoven University of Technology, Eindhoven,Netherlands, 2002.

[12]E. F. Barbosa, M. L. Fioravanti, E. Y. Nakagawa, and J. C. Maldonado, "Towards the Establishment of a Reference Architecture for Developing Learning Environments," in SEKE, 2013, pp. 350-355.

[13]B. Kitchenham and P. Brereton, "A systematic review of systematic review process research in software engineering," Inf. Softw. Technol., vol. 55, no. 12, pp. 2049-2075, 2013.

[14]T. F. Erl, "Service oriented architecture (SOA) modeling." Google Patents, 2015.

[15]N. F. Duarte Filho and E. F. Barbosa, "A service-oriented reference architecture for mobile learning environments," in Frontiers in Education Conference (FIE), 2014 IEEE, 2014, pp. $1-8$.

[16]N. F. Duarte Filho and E. F. Barbosa, "Defining a Set of Architectural Requirements for Service-Oriented Mobile Learning Environments.," Assoc. Dev. Inf. Soc., 2014.

[17] V. R. Basili, "Applying the Goal/Question/Metric paradigm in the experience factory," Softw. Qual. Assur. Meas. A Worldw. Perspect., pp. 21-44, 1993. 